\title{
Molecular Communication via Subdiffusion with a Spherical Absorbing Receiver
}

\author{
Shuai Huang, Lin Lin, Juan Xu, Weisi Guo, Hao Yan
}

\begin{abstract}
In molecular communication (MC), the motion of information molecules in the medium is usually described by the Brownian motion and governed by the Fick's laws. However, there are some potential scenarios of MC where the kinetics of information molecules is non-Fickian. In this letter, we investigate one of this kind of MC. The manner of information molecules in the channel is subdiffusion. A three-dimensional MC system with a spherical absorbing receiver is considered. The subdiffusion channel is analyzed. The closed-form expressions of the first hitting probability and its peak time are given. Furthermore, we investigate the performance of MC by timing and amplitude modulation schemes in a subdiffusion channel. The error probability for both modulation schemes is analyzed.
\end{abstract}

Index Terms-Molecular communication, anomalous diffusion, error probability.

\section{INTRODUCTION}

$\mathbf{M}$ OLECULAR communication (MC) is a paradigm that small particles are used for information carrier [1]. In literature, diffusion-based MC has been investigated widely where the propagation of information molecules in the channel is diffusion. The diffusion scheme adopted in MC is usually Fickian which obeys Fick's laws. However, there are some scenarios where the diffusion process of a molecule can not be categorized as this kind of diffusion. For example, the diffusion observed by experiments in crowded, heterogeneous, or complex structure systems is non-Fickian [2], which is also named anomalous diffusion.

In literature, $\mathrm{MC}$ via anomalous diffusion was investigated in [3] firstly where 1-D communication channel is considered. This work was developed to a connectivity problem with a random time constraint in a 1-D nanonetwork [4]. Analogously, in [5], the authors focused on MC by anomalous diffusion in a 2-D stochastic nanonetwork. The 3-D MC in an anomalous diffusion channel was considered in [6]. The authors analyzed the performance of a concentration-encoded MC system where the manner of information molecules is subdiffusion.

This work was supported in part by National Natural Science Foundation, China (61971314), in part by Natural Science Foundation of Shanghai (19ZR1426500), in part by Science and Technology Commission of Shanghai Municipality (19510744900), and in part by the EU Horizon 2020 Programme (792799). (Corresponding author: Lin Lin)

S. Huang, L. Lin and J. Xu are with the College of Electronics and Information Engineering, Tongji University, Shanghai 201804, China. (e-mail: shuailuck@163.com; fxlinlin@tongji.edu.cn; jxujuan@tongji.edu.cn).

W. Guo is with Cranfield University, MK43 0AL, U.K. (e-mail: weisi.guo@ cranfield.ac.uk).

H. Yan is with the School of Electronic, Information and Electrical Engineering, Shanghai Jiao Tong University, Shanghai 200240, China (email: yan_hao@sjtu.edu.cn).
In this letter, similar to [6], we investigate 3-D MC in a subdiffusive channel. The propagation scheme of information molecules is subdiffusion, one kind of anomalous diffusion. This kind of diffusion process is common in some classical applications of MC. For instance, in living cells, proteins which are searching for specific DNA target sites undergo subdiffusion due to the crowding and caging, geometrical traps and energetic barriers [7]. As another example, for the particles in the bulk, one can consider them to be governed by the subdiffusion [8]. Unlike the work in [6] where the receiver model is passive, in this letter, a spherical absorbing receiver is adopted. Compared with the former, the extra boundary condition is introduced by the absorbing receiver. To the best of our knowledge, this is the first investigation of $\mathrm{MC}$ with an absorbing receiver in a 3-D subdiffusive channel. The main contributions of this letter are as follows:

1) Channel analysis: We derive the channel instantaneous response, i.e, the first hitting probability. Furthermore, the exact expression of the peak time is derived, which has a great significance for the choice of symbol duration for a MC system.

2) Error probability: We investigate the error performance of MC in a subdiffusion channel with two different modulation methods, i.e., timing and amplitude modulation schemes. The closed-form expression of the error probability for the timing modulation is derived.

\section{SYSTEM MODEL}

The complete 3-D MC system considered in this letter consists of a transmitter-receiver pair, a subdiffusion molecular channel, and information-carrying messenger molecules. The transmitter is a point source which is located at distance $r_{0}$ from the center of the receiver. The receiver is a 3-D sphere of radius $a_{\mathrm{rx}}$ with fully absorbing boundaries [9]. That is, the receiver is covered with selective independent receptors, which are only sensitive to a single type of information molecule. Every information molecule colliding with the surface of the sphere is absorbed by the receiver body and removed from the communication environment. Fig. 1 depicts a diagram of the system.

Unlike the Fickian diffusion channel, the propagation scheme of information molecules is subdiffusion. This kind of diffusion usually appears in crowded environment. Experiments on particle motion in living cells and in biological and artificial membranes have shown that the diffusion in such environment is often subdiffusion [10], i.e., it does not correspond to the particle's mean squared displacement 


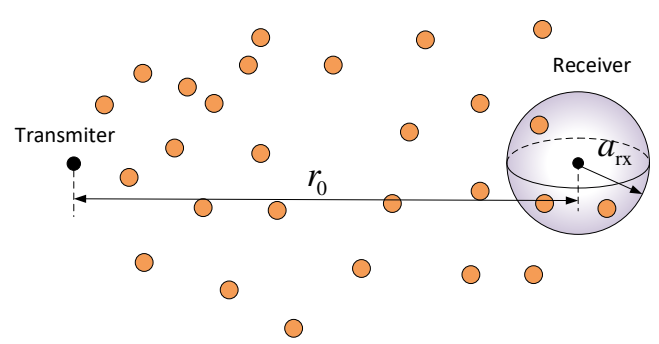

Fig. 1. System model. The propagation scheme of information molecule is subdiffusion.

growing linearly in time, $<r^{2}(t)>\propto t$, as predicted by Fick's theory of diffusion, but follows another fractionalpower pattern $<r^{2}(t)>\propto t^{\gamma}$, with an exponent $\gamma$ between 0 and 1 . The subdiffusion manner of information molecule can be described by the following time-fractional partial differential equation [6, Eq. (10)]:

$$
\frac{\partial}{\partial t} w\left(r, t ; r_{0}\right)={ }_{0} D_{t}^{1-\gamma}\left[D \nabla_{r}^{2} w\left(r, t ; r_{0}\right)\right] .
$$

In (1), $w\left(r, t ; r_{0}\right)$ is the probability distribution of a molecule in time-space domain. $D$ is the diffusion constant and $\nabla_{r}^{2}$ is the Laplacian operator on $r .{ }_{0} D_{t}^{1-\gamma}$ is the Riemann-Liouvile operator defined as [6, Eq. (8)]

$$
{ }_{0} D_{t}^{1-\gamma}[f(r, t)]=\frac{1}{\Gamma(\gamma)} \frac{\partial}{\partial t} \int_{0}^{t} \frac{f(r, \tau)}{(t-\tau)^{1-\gamma}} \mathrm{d} \tau, 0<\gamma<1 .
$$

Here $\Gamma(\cdot)$ denotes the gamma function. Note that (1) will reduce to Fick's second law when $\gamma=1$ since

$$
{ }_{0} D_{t}^{0}[f(r, t)]=f(r, t) .
$$

In addition to the time-fractional subdiffusion equation, we should define the initial and the boundary conditions obeying the model in Fig. 1. These conditions have been given in [9].

\section{Channel Characteristics}

In this section, the subdiffusion $\mathrm{MC}$ channel is investigated. First we focus on the absorption probability $K\left(t ; r_{0}\right)$, which is the probability that a molecule is absorbed by the receiver in $[0, t]$ after it is released by the transmitter at time $t=0$. Since $w\left(r, t ; r_{0}\right)$ is the probability distribution of a molecule in time-space domain, $K\left(t ; r_{0}\right)$ can be calculated by

$$
K\left(t ; r_{0}\right)=1-\int_{a_{\text {гх }}}^{\infty} 4 \pi r^{2} w\left(r, t ; r_{0}\right) \mathrm{d} r .
$$

One can understand (4) by regarding the integral in (4) as the survival probability of a molecule after it is released, which has been derived in [11, Eq. (55)] by the Laplacian transform. Here we use the conclusion of [11] directly to obtain the expression of $K\left(t ; r_{0}\right)$ :

$$
\begin{aligned}
K\left(t ; r_{0}\right) & =\frac{a_{\mathrm{rx}}}{r_{0}} H_{11}^{10}\left[\frac{r_{0}-a_{\mathrm{rx}}}{\sqrt{D t^{\gamma}}} \mid \begin{array}{c}
(1, \gamma / 2) \\
(0,1)
\end{array}\right], \\
K^{*}\left(s ; r_{0}\right) & =\frac{1}{s} \frac{a_{\mathrm{rx}}}{r_{0}} \exp \left(\left(a_{\mathrm{rx}}-r_{0}\right) \sqrt{s^{\gamma} / D}\right) .
\end{aligned}
$$

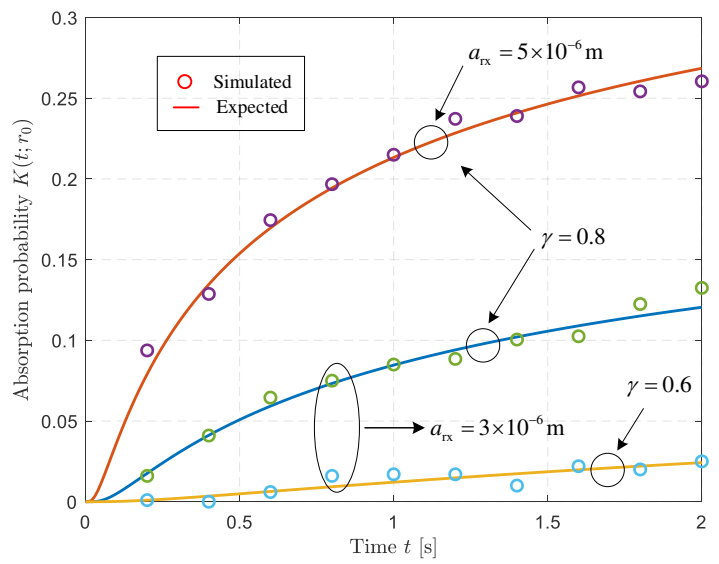

Fig. 2. The absorption probability $K\left(t ; r_{0}\right)$.

In (5), $H_{p, q}^{m, n}(\cdot)$ is Fox's $\mathrm{H}$ function [12]. $K^{*}\left(s ; r_{0}\right)$ is the Laplacian transform of $K\left(t ; r_{0}\right)$ with respect to time $t$. Note that when $t \rightarrow \infty$, the absorption probability $K\left(t ; r_{0}\right)$ has a limit:

$$
\lim _{t \rightarrow \infty} K\left(t ; r_{0}\right)=\lim _{s \rightarrow 0} s K^{*}\left(s ; r_{0}\right)=\frac{a_{\mathrm{rx}}}{r_{0}} .
$$

The result in (7) agrees with the common sense, i.e., the larger receiver provides more possibilities for a molecule absorbed. On the other hand, if $\gamma=1$, (5) is given by

$$
\begin{aligned}
\left.K\left(t ; r_{0}\right)\right|_{\gamma=1} & =\frac{a_{\mathrm{rx}}}{r_{0}} H_{11}^{10}\left[\frac{r_{0}-a_{\mathrm{rx}}}{\sqrt{D t}} \mid \begin{array}{c}
(1,1 / 2) \\
(0,1)
\end{array}\right] \\
& \stackrel{(a)}{=} \frac{a_{\mathrm{rx}}}{r_{0}}\left[1+\sum_{k=1}^{\infty} \frac{(-z)^{k}}{k ! \Gamma\left(1-\frac{1}{2} k\right)}\right] .
\end{aligned}
$$

In (8), $z=\left(r_{0}-a_{\mathrm{rx}}\right) / \sqrt{D t}$. The equality (a) holds due to the power series expansion of the Fox's H function [13]. Then, (8) can be rewritten as

$$
\begin{aligned}
\left.K\left(t ; r_{0}\right)\right|_{\gamma=1} & =\frac{a_{\mathrm{rx}}}{r_{0}}\left[1+\sum_{k=1}^{\infty} \frac{2 \sin \left(\frac{k}{2} \pi\right) \Gamma\left(1+\frac{k}{2}\right)(-z)^{k}}{k \cdot k ! \pi}\right] \\
& =\frac{a_{\mathrm{rx}}}{r_{0}}\left[1-\sum_{n=0}^{\infty} \frac{(-1)^{n} z^{2 n+1} \Gamma\left(n+\frac{1}{2}\right)}{(2 n+1) ! \pi}\right] \\
& =\frac{a_{\mathrm{rx}}}{r_{0}}\left[1-\frac{2}{\sqrt{\pi}} \sum_{n=0}^{\infty} \frac{(-1)^{n}\left(\frac{1}{2} z\right)^{2 n+1}}{n !(2 n+1)}\right]
\end{aligned}
$$

where the series in (9) is the expansion of the error function. Thus, the absorption probability for Fickian diffusion $(\gamma=1)$ is given by

$$
\left.K\left(t ; r_{0}\right)\right|_{\gamma=1}=\frac{a_{\mathrm{rx}}}{r_{0}} \operatorname{erfc} \frac{z}{2}=\frac{a_{\mathrm{rx}}}{r_{0}} \operatorname{erfc}\left[\frac{r_{0}-a_{\mathrm{rx}}}{\sqrt{4 D t}}\right],
$$

which is same with the result in [9, Eq. (23)].

Fig. 2 shows the comparison between the simulated and expected absorption probabilities $K\left(t ; r_{0}\right)$, denoted by the circle and the solid line, respectively. The simulations are particle based. The number of molecules released is $N=10^{3}$. 
The receiver counts the number of molecules absorbed during the time slot $[0, t]$, denoted by $N_{\mathrm{ab}}$. Then the ratio $N_{\mathrm{ab}} / N$ is the simulated absorption probability. For characterizing the movement of each molecule in the channel, we use a Monte Carlo method connected to the continuous-time random walks (CTRW) with Gaussian and Lévy distributions. The relation between the CTRW and the subdiffusion can be found in [13, Section II]. The simulation parameters are set as $r_{0}=10^{-5} \mathrm{~m}$, $\sigma^{2}=2 \times 10^{-14} \mathrm{~m}^{2}, \tau=10^{-5}$ s. $\sigma, \tau$ are scaling parameters for the space and time variables, which are related to the diffusion coefficient, defined as $D=\sigma^{2} / 2 \Gamma(1-\gamma) \tau^{\gamma}$ by taking the limit $\sigma^{2} \rightarrow 0$ and $\tau \rightarrow 0$. As shown in Fig. 2 , the simulated results are in agreement with the theoretic prediction. Furthermore, we observe that it is more easier for information molecule to be absorbed when the size of receiver and the value of $\gamma$ are bigger.

The absorption probability $K\left(t ; r_{0}\right)$ characterizes the subdiffuion channel over time. Generally, it is more intuitive to use the instantaneous response to describe the channel. For a subdiffusion channel with a spherical absorbing receiver, its instantaneous response is the first hitting probability, defined as [9, Eq. (23)]

$$
f_{\text {hit }}\left(t ; r_{0}\right)=\frac{\partial}{\partial t} K\left(t ; r_{0}\right) .
$$

By setting $x=\left(r_{0}-a_{\mathrm{rx}}\right) / \sqrt{D t^{\gamma}}$, (11) can be stated as

$$
f_{\text {hit }}\left(t ; r_{0}\right)=\frac{a_{\mathrm{rx}}}{r_{0}} \frac{\partial x}{\partial t} \frac{\partial}{\partial x} H_{11}^{10}\left[x \mid \begin{array}{c}
(1, \gamma / 2) \\
(0,1)
\end{array}\right] \text {. }
$$

Using the Mellin-Barnes integral representation of Fox's $\mathrm{H}$ function [12], (12) is given by

$$
f_{\text {hit }}\left(t ; r_{0}\right)=\frac{a_{\mathrm{rx}}}{r_{0}} \frac{\partial x}{\partial t} \frac{\partial}{\partial x}\left[\frac{1}{2 \pi i} \int_{L} \frac{\Gamma(s)}{\Gamma\left(1+\frac{\gamma}{2} s\right)} x^{-s} \mathrm{~d} s\right] \text {. }
$$

The order of differentiation and integration can be changed, see [14, Eq. (2.5)], then (13) can be calculated by

$$
\begin{aligned}
f_{\text {hit }}\left(t ; r_{0}\right) & =-\frac{a_{\mathrm{rx}}}{r_{0}} x^{-1} \frac{\partial x}{\partial t} \frac{1}{2 \pi i} \int_{L} \frac{\Gamma(s+1)}{\Gamma\left(1+\frac{\gamma}{2} s\right)} x^{-s} \mathrm{~d} s \\
& =-\frac{a_{\mathrm{rx}}}{r_{0}} x^{-1} \frac{\partial x}{\partial t} H_{11}^{10}\left[\begin{array}{c}
(1, \gamma / 2) \\
(1,1)
\end{array}\right] .
\end{aligned}
$$

Finally, the first hitting probability function can be written as

$$
f_{\text {hit }}\left(t ; r_{0}\right)=\frac{a_{\mathrm{rx}} \gamma}{2 r_{0} t} H_{11}^{10}\left[\frac{r_{0}-a_{\mathrm{rx}}}{\sqrt{D t^{\gamma}}} \mid \begin{array}{c}
(1, \gamma / 2) \\
(1,1)
\end{array}\right] .
$$

It can be proven that (15) will reduce to [9, Eq. (22)] when $\gamma=1$.

Similar to [9], the peak time of the first hitting probability $f_{\text {hit }}\left(t ; r_{0}\right)$ is investigated in the remainder of this section. However, it is difficult to obtain the maximum of $f_{\text {hit }}\left(t ; r_{0}\right)$ by using the first derivative test. Inspired by [13], the pulse peak time $t_{\text {peak }}$ can be found by the asymptotic behavior of $f_{\text {hit }}\left(t ; r_{0}\right)$ for small value of $t$. According to [13, Eq. (3.35)], the Fox's H function in (19) has the asymptotic expansion as

$$
\begin{aligned}
H_{11}^{10}\left[\frac{r_{0}-a_{\mathrm{rx}}}{\sqrt{D t^{\gamma}}} \mid \begin{array}{c}
(1, \gamma / 2) \\
(1,1)
\end{array}\right] \sim\left(\frac{2}{\pi \gamma(2-\gamma)}\right)^{1 / 2}\left(\frac{\gamma}{2}\right)^{\frac{\gamma}{2(2-\gamma)}} \\
\quad \times z^{1 /(2-\gamma)} \exp \left(-\frac{2-\gamma}{2}\left(\frac{\gamma}{2}\right)^{\gamma /(2-\gamma)} z^{2 /(2-\gamma)}\right) .
\end{aligned}
$$

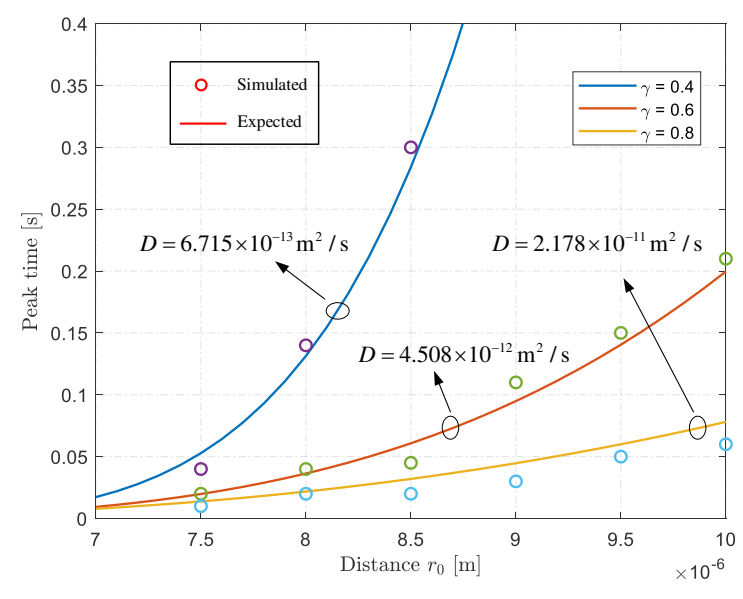

Fig. 3. The pulse peak time in (19).

Substituting this in the expression for $f_{\text {hit }}\left(t ; r_{0}\right)$ in (15), we can obtain for small $t$

$$
f_{\text {hit }}\left(t ; r_{0}\right) \sim \frac{r}{t^{(4-\gamma) /(4-2 \gamma)}} \exp \left(-\frac{d}{t^{\gamma /(2-\gamma)}}\right),
$$

where

$$
\begin{aligned}
& r=\left(\frac{2 \gamma}{\pi(2-\gamma)}\right)^{1 / 2}\left(\frac{\gamma}{2}\right)^{\frac{\gamma}{2(2-\gamma)}}\left(\frac{r_{0}-a_{\mathrm{rx}}}{\sqrt{D}}\right)^{1 /(2-\gamma)} \\
& d=\frac{2-\gamma}{2}\left(\frac{\gamma}{2}\right)^{\gamma /(2-\gamma)}\left(\frac{r_{0}-a_{\mathrm{rx}}}{\sqrt{D}}\right)^{2 /(2-\gamma)}
\end{aligned}
$$

We can determine the $t$ value where $f_{\text {hit }}\left(t ; r_{0}\right)$ attains its maximum value from the above expression (17):

$$
t_{\text {peak }}=\frac{\gamma}{2}\left(\frac{2-\gamma}{4-\gamma}\right)^{\frac{2-\gamma}{\gamma}}\left(\frac{r_{0}-a_{\mathrm{rx}}}{\sqrt{D}}\right)^{\frac{2}{\gamma}} .
$$

Fig. 3 presents the distance $r_{0}$ versus the pulse peak time $t_{\text {peak }}$ for different $\gamma$ values. Simulation results denoted by the circle, are particle based. The number of molecule released is $5 \times 10^{4}$. Similar to the simulations in Fig. 2, the CTRW with same parameters, i.e., $\sigma$ and $\tau$, is used to characterize the subdiffusion manner of each molecule. As shown in Fig. 3 , simulation results validate the analytical expression of the pulse peak time. Furthermore, note that the peak time is smaller for larger values of $\gamma$, This is due to the fact that the diffusion coefficient $D=\sigma^{2} / 2 \Gamma(1-\gamma) \tau^{\gamma}$ is larger as $\gamma$ increases.

\section{ERROR PERFORMANCE}

In this section, we investigate the error performance of $\mathrm{MC}$ in a subdiffusion channel. The transmitter sends a binary message sequence to the receiver. The time interval for bit transmission is $T_{\mathrm{b}}$. Let $b_{i} \in\{0,1\}$ denotes the bit transmitted in the $i$ th bit interval. Two modulation schemes-i.e., timing and amplitude modulation, are presented in the remainder of this section. 


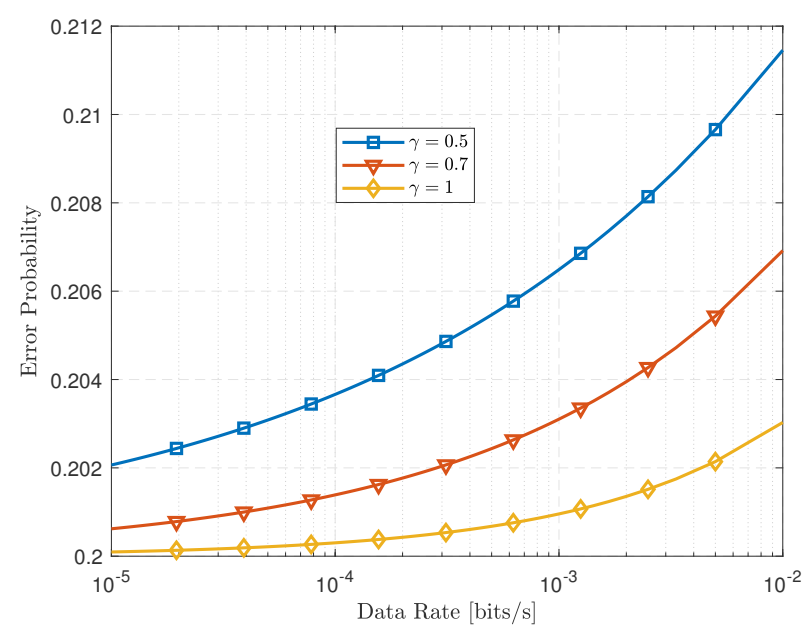

Fig. 4. Error probability in (21) for the timing modulation. The parameters in $(21)$ are defined as $a_{\mathrm{rx}}=6 \times 10^{-6} \mathrm{~m}, r_{0}=10^{-5} \mathrm{~m}$, and $\xi_{\mathrm{th}}=1 / 2 T_{\mathrm{b}}$.

\section{A. Timing Modulation}

For timing modulation, information is encoded into the molecule release time. The transmitter releases information molecule at the start of a symbol slot if $b_{i}=0$ while the emission time is the middle of a symbol slot if $b_{i}=1$. In other words, $X \in\left\{0, T_{\mathrm{b}} / 2\right\}$ where $X$ denotes the release time. For simplicity, it is assumed that the channel is memoryless so that ISI is negligible. Let $Y$ be the arrival time ${ }^{1}$, i.e., the instant when the released molecule is absorbed by the receiver. Then, decision is given by

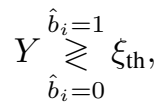

where $\hat{b}_{i}$ is the estimate of $b_{i}$ and $\xi_{\text {th }}$ is the detection threshold. Considering a symmetric bit input, the error probability $P_{\mathrm{b}}$ for this timing modulation of $\mathrm{MC}$ in a subdiffusion channel is given by

$$
\begin{aligned}
P_{\mathrm{b}} & =\frac{1}{2} \mathbb{P}\left(Y \geqslant \xi_{\text {th }} \mid X=0\right)+\frac{1}{2} \mathbb{P}\left(Y<\xi_{\text {th }} \mid X=\frac{T_{\mathrm{b}}}{2}\right) \\
& =\frac{1}{2}\left(1-K\left(\xi_{\text {th }} ; r_{0}\right)\right)+\frac{1}{2} K\left(\xi_{\text {th }}-\frac{T_{\mathrm{b}}}{2} ; r_{0}\right) .
\end{aligned}
$$

Fig. 4 depicts the error probability $P_{\mathrm{b}}$ in (21). The Fox's $\mathrm{H}$ function is calculated by its Mellin-Barnes integral representation, see [15] for the implementation of Fox's H function by MATLAB. As shown in the figure, the subdiffusion increases the error probability compared with the Fickian diffusion, i.e., $\gamma=1$. The reason behind this phenomenon is the anomalous diffusion has larger dispersion [3]. Note that the error probability $P_{\mathrm{b}}$ will never approach to zero no matter how small the data rate $\left(1 / T_{\mathrm{b}}\right)$ is. That is due to the fact that the absorption probability has a limit, see (7).

\footnotetext{
${ }^{1}$ According to (7), there is a positive probability that the transmitted molecule never arrives. In this case, it is assumed that the arrive time $Y$ is equal to infinity.
}

\section{B. Amplitude Modulation}

For the amplitude modulation, information is modulated by the number of molecules released by the transmitter at the beginning of a symbol slot. For simplicity, the On-Off Keying $(\mathrm{OOK})$ is adopted where the number of molecules to be released is $Q$ for $b_{i}=1$, and 0 for $b_{i}=0$. The receiver counts the number of molecules absorbed in the current symbol slot as the received signal, and compares this value with a detection threshold. Unlike the timing modulation, ISI is considered here. Let $\boldsymbol{b}_{i}$ denote the transmitted binary sequence, i.e., $\boldsymbol{b}_{i}=\left[b_{1}, \cdots, b_{i}\right]$. The received signal $Y_{i}$ at the $i$ th time slot is a Gaussian variable which is given by

$$
Y_{i} \sim \mathcal{N}\left(\mu_{i}, \sigma_{i}^{2}\right)
$$

In (22), $\mu_{i}, \sigma_{i}^{2}$ are the mean and variance of $Y_{i}$, respectively. Due to the effect of ISI and noise, $\mu_{i}, \sigma_{i}^{2}$ are given by

$$
\mu_{i}=Q \boldsymbol{b}_{i} \cdot \boldsymbol{p}_{i}^{T}, \sigma_{i}^{2}=Q \boldsymbol{b}_{i} \cdot \operatorname{vdiag}\left\{\boldsymbol{p}_{i}^{T}\left(\mathbf{1}-\boldsymbol{p}_{i}\right)\right\}+\sigma_{c}^{2} .
$$

In (23), $\operatorname{vdiag}\{\boldsymbol{A}\}$ is a column vector which contains the diagonal entries of matrix $\boldsymbol{A} . \sigma_{c}^{2}$ is the variance of a zeromean white Gaussian noise. $\boldsymbol{p}_{i}=\left[p_{i}, p_{i-1}, \cdots, p_{1}\right]$ is used to characterize the effect of ISI, which is a vector including the absorption probabilities $p_{m}, m=1, \cdots, i$, defined as

$$
p_{m}= \begin{cases}K\left(m T_{\mathrm{b}} ; r_{0}\right)-K\left((m-1) T_{\mathrm{b}} ; r_{0}\right) & \text { if } m>1 \\ K\left(T_{\mathrm{b}} ; r_{0}\right) & \text { if } m=1\end{cases}
$$

The detection threshold in the $i$ th bit interval is assumed as $\xi_{i}$. Then, decision is given by

$$
Y_{i} \stackrel{\hat{b}_{i}=0}{\gtrless} \xi_{i}
$$

The optimal threshold $\xi_{i}^{*}$ can be calculated by forming the likelihood ratio test

$$
\xi_{i}^{*}=\underset{y_{i}}{\arg \min }\left|1-\frac{f_{Y_{i}}\left(y_{i} \mid \boldsymbol{b}_{i-1}, b_{i}=1\right)}{f_{Y_{i}}\left(y_{i} \mid \boldsymbol{b}_{i-1}, b_{i}=0\right)}\right|,
$$

where $f_{Y_{i}}(\cdot)$ denotes the probability density function of the Gaussian distributed $Y_{i}$. Note that it is necessary for the receiver to keep the sequence history $\boldsymbol{b}_{i-1}$ for the sake of calculation of $\xi_{i}^{*}$. In other words, the detector has memory. The error probability at $i$ th bit interval given $\boldsymbol{b}_{i-1}$ is given by

$$
\left.P_{\mathrm{b}}^{i}\right|_{\boldsymbol{b}_{i-1}}=\frac{1}{2} Q\left(\frac{\xi_{i}^{*}-\mu_{i 0}}{\sigma_{i 0}}\right)+\frac{1}{2} Q\left(\frac{\mu_{i 1}-\xi_{i}^{*}}{\sigma_{i 1}}\right),
$$

where $\left\{\mu_{i 0}, \sigma_{i 0}\right\},\left\{\mu_{i 1}, \sigma_{i 1}\right\}$ are the parameter sets of $Y_{i}$ given $b_{i}=0$ and $b_{i}=1$, respectively. For the memoryless detector, the optimal threshold $\xi_{i}^{*}$ can be calculated by [16, Algorithm 1].

Fig. 5(a) shows the error probability in (27) given the most serious ISI effect, i.e., the sequence of ISI is $\boldsymbol{b}_{i-1}=$ $[1,1, \cdots, 1]$. The parameters are set as $a_{\mathrm{rx}}=5 \times 10^{-6} \mathrm{~m}$, $r_{0}=10^{-5} \mathrm{~m}, Q=200, \sigma^{2}=10^{-12} \mathrm{~m}^{2}, \tau=10^{-5} \mathrm{~s}$, and $\sigma_{c}^{2}=2$. Note that the error probability is smaller for larger values of $\gamma$. The reason behind the phenomenon can be found in Fig. 2. As shown in Fig. 2, the absorption probability $K\left(t ; r_{0}\right)$ grows faster for large value of $\gamma$ in the beginning. 


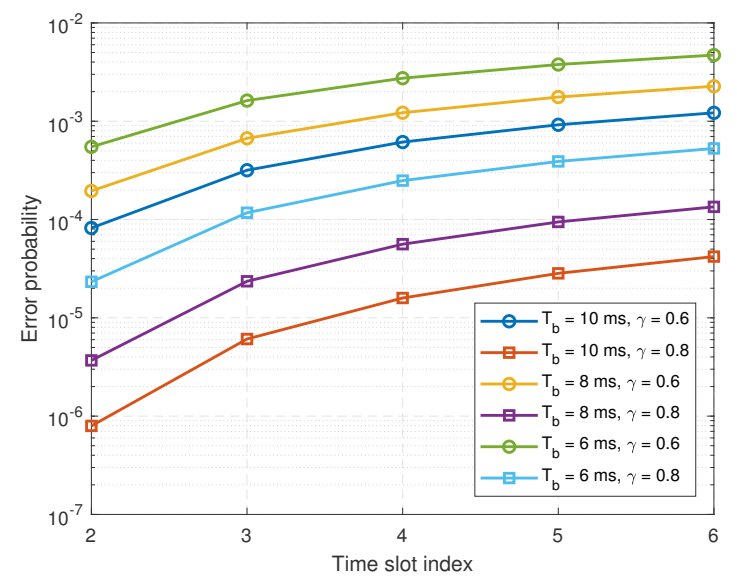

(a) Error probability $\left.P_{\mathrm{b}}^{i}\right|_{\boldsymbol{b}_{i-1}}$ for different values of $T_{\mathrm{b}}$ and $\gamma$.

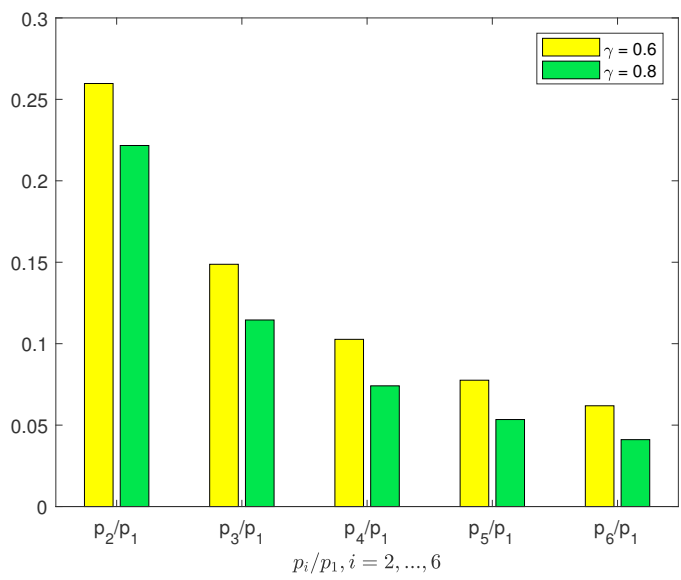

(b) The ISI vector $\boldsymbol{p}_{i}$ for different values of $\gamma$.

Fig. 5. The error probability in (27) affected by the worst ISI based on amplitude modulation.

In other words, $p_{1}=K\left(T_{\mathrm{b}} ; r_{0}\right)$ is bigger for large value of $\gamma$, which is beneficial to the information transmission. Furthermore, ISI is more serious when $\gamma=0.6$ from the bar graph in Fig. 5(b).

\section{CONCLUSION}

In this letter, we investigate $\mathrm{MC}$ in a 3-D subdiffusion channel with an absorbing receiver. The subdiffusion channel is analyzed. The formulas of the first hitting probability and its peak time are derived. Furthermore, the error performance for the timing and amplitude modulation is considered, respectively. The simulation results show that the larger value of $\gamma$ leads to the better performance. For the timing modulation discussed in Section IV, note that only an information molecule is released by the transmitter and the ISI is not considered in the demodulation process. It will be our future work to improve the performance of this timing modulation scheme.

\section{REFERENCES}

[1] T. Nakano, A. W. Eckford, and T. Haraguchi, Molecular Communication, 1st ed. Cambridge, U.K.: Cambridge Univ. Press, 2013.

[2] R. Metzler and J. Klafter, "The random walk's guide to anomalous diffusion: a fractional dynamics approach," Phys. Rep., vol. 339, no. 1, pp. 1-77, Dec. 2000.

[3] T. N. Cao, D. P. Trinh, Y. Jeong, and H. Shin, "Anomalous diffusion in molecular communication," IEEE Commun. Lett., vol. 19, no. 10, pp. 1674-1677, Oct. 2015.

[4] D. P. Trinh, Y. Jeong, and H. Shin, "Connectivity in molecular communication with random time constraints," IEEE Access, vol. 7, pp. 113 121-113 130, Aug. 2019.

[5] D. P. Trinh, Y. Jeong, H. Shin, and M. Z. Win, "Molecular communication with anomalous diffusion in stochastic nanonetworks," IEEE Trans. Commun., vol. 67, no. 12, pp. 8378-8393, Dec 2019.

[6] M. U. Mahfuz, D. Makrakis, and H. T. Mouftah, "Concentrationencoded subdiffusive molecular communication: Theory, channel characteristics, and optimum signal detection," IEEE Trans. Nanobiosci., vol. 15 , no. 6, pp. 533-548, Sep. 2016.

[7] M. Coppey, O. Bénichou, R. Voituriez, and M. Moreau, "Kinetics of target site localization of a protein on dna: a stochastic approach," Biophys. J., vol. 87, no. 3, pp. 1640-1649, Sep. 2004.

[8] E. K. Lenzi, D. S. Vieira, M. K. Lenzi, G. Goncalves, and D. P. Leitoles, "Solutions for a fractional diffusion equation with radial symmetry and integro-differential boundary conditions," Thermal Science, vol. 19, no. 1, pp. S1-S6, 2015.
[9] H. B. Yilmaz, A. C. Heren, T. Tugcu, and C.-B. Chae, "Threedimensional channel characteristics for molecular communications with an absorbing receiver," IEEE Commun. Lett., vol. 18, no. 6, pp. 929932, Apr. 2014

[10] P. R. Smith, I. E. Morrison, K. M. Wilson, N. Fernndez, and R. J. Cherry, "Anomalous diffusion of major histocompatibility complex class i molecules on hela cells determined by single particle tracking," Biophys. J., vol. 76, no. 6, pp. 3331-3344, Jun. 1999.

[11] S. B. Yuste and K. Lindenberg, "Subdiffusive target problem: Survival probability," Phys Rev E Stat Nonlin Soft Matter Phys, vol. 76, no. 5 Pt 1, p. 051114, 2007.

[12] A. M. Mathai, R. K. Saxena, and H. J. Haubold, The H-Function: Theory and Applications. Springer, 2010.

[13] G. Rangarajan and M. Ding, "Anomalous diffusion and the first passage time problem," Phys. Rev. E, vol. 62, no. 1, p. 120, Jul. 2000.

[14] P. Anandani, "On some recurrence formulae for the h-function," Annales Polonici Mathematici, vol. 21, no. 2, pp. 113-117, 1969.

[15] K. P. Peppas, F. Lazarakis, A. Alexandridis, and K. Dangakis, "Simple, accurate formula for the average bit error probability of multipleinput multiple-output free-space optical links over negative exponential turbulence channels," Optics Lett., vol. 37, no. 15, pp. 3243-3245, Aug. 2012.

[16] B. Tepekule, A. E. Pusane, H. B. Yilmaz, C.-B. Chae, and T. Tugcu, "Isi mitigation techniques in molecular communication," IEEE Trans. Mol. Biol. Multi-Scale Commun., vol. 1, no. 2, pp. 202-216, Jun. 2015. 
2020-06-09

\title{
Molecular communication via
}

\section{subdiffusion with a spherical absorbing receiver}

\author{
Huang, Shuai
}

IEEE

Huang S, Lin L, Xu J, et al., (2020) Molecular communication via subdiffusion with a spherical absorbing receiver. IEEE Wireless Communications, Volume 9, Issue 10, October 2020, pp. 1682-1686 https://doi.org/10.1109/LWC.2020.3001148

Downloaded from Cranfield Library Services E-Repository 